\title{
SPANDx: a genomics pipeline for comparative analysis of large haploid whole genome re-sequencing datasets
}

\author{
Derek S Sarovich ${ }^{1 *}$ and Erin P Price ${ }^{1,2}$
}

\begin{abstract}
Background: Next-generation sequencing (NGS) is now a commonplace tool for molecular characterisation of virtually any species of interest. Despite the ever-increasing use of NGS in laboratories worldwide, analysis of whole genome re-sequencing (WGS) datasets from start to finish remains nontrivial due to the fragmented nature of NGS software and the lack of experienced bioinformaticists in many research teams.

Findings: We describe SPANDx (Synergised Pipeline for Analysis of NGS Data in Linux), a new tool for high-throughput comparative analysis of haploid WGS datasets comprising one through thousands of genomes. SPANDx consolidates several well-validated, open-source packages into a single tool, mitigating the need to learn and manipulate individual NGS programs. SPANDx incorporates BWA for alignment of raw NGS reads against a reference genome or pan-genome, followed by data filtering, variant calling and annotation using Picard, GATK, SAMtools and SnpEff. BEDTools has also been included for genetic locus presence/absence (P/A) determination to easily visualise the core and accessory genomes. Additional SPANDx features include construction of error-corrected single-nucleotide polymorphism (SNP) and insertion-deletion matrices, and P/A matrices, to enable user-friendly visualisation of genetic variants. The SNP matrices generated using VCFtools and GATK are directly importable into PAUP*, PHYLIP or RAxML for downstream phylogenetic analysis. SPANDx has been developed to handle NGS data from Illumina, Ion Personal Genome Machine (PGM) and 454 platforms, and we demonstrate that it has comparable performance across Illumina MiSeq/HiSeq2000 and Ion PGM data.
\end{abstract}

Conclusion: SPANDx is an all-in-one tool for comprehensive haploid WGS analysis. SPANDx is open source and is freely available at: http://sourceforge.net/projects/spandx/.

Keywords: NGS, Haploid, Pipeline, Comparative genomics, Illumina, Ion PGM, Variant calling, SNP, Indel, Phylogeny

\section{Background}

The development of the first massively parallel nextgeneration sequencing (NGS) platform in 2005 [1] forever changed the medical and biological research landscape. A decade on, NGS technologies are now being routinely used for myriad purposes including whole-genome re-sequencing (WGS), genome-wide association studies, de novo- and re-assemblies, amplicon re-sequencing, polymorphism discovery, non-coding and coding RNA characterisation (RNA-seq), methylation studies (Methyl-seq) and protein-DNA interactions

\footnotetext{
* Correspondence: derek.sarovich@menzies.edu.au

${ }^{1}$ Global and Tropical Health Division, Menzies School of Health Research, Charles Darwin University, PO Box 41096, Casuarina 0811, NT, Australia Full list of author information is available at the end of the article
}

(ChIP-seq). The popularity of NGS has led to a rapid decrease in operating and reagent costs that have outstripped the "Moore's law" paradigm, a common yardstick for measuring technological success based on computational hardware speed (http://www.genome.gov/sequencingcosts/). This plummeting cost has been brought about by major technological improvements and increased competition in the NGS platform market. Given continuing improvements in cost-effectiveness and versatility of NGS in molecular biology research, it is not surprising that NGS has become a mainstay in both small and large research laboratories across the globe.

The desire to answer important medical or biological questions using NGS, and in particular WGS, has concurrently driven the development of analysis tools 
designed to efficiently and accurately decode these vast volumes of nucleic acid data. However, analysis has been unable to keep pace with the volume of data being generated. Challenges to NGS data management and analysis include computation and storage availability and scalability, data sharing and privacy issues, NGS software costs and the requirement for bioinformaticists skilled in designing, programming and running complex analysis pipelines [2]. The technical difficulty and fragmented nature of NGS software, particularly for large-scale WGS analyses involving more than a handful of genomes, mean that comprehensive analyses remain out of reach for many researchers. In addition, the lack of transparent, publicly available and standardised NGS pipelines has potentially led to non-validated variant outputs being reported and perpetuated in the literature.

To address these issues, we have developed SPANDx (Synergised Pipeline for Analysis of NGS Data in Linux). SPANDx is an open-source, high-throughput, comparative genomic analysis tool for haploid organisms that integrates well-validated, open-source programs into a single program, thereby simplifying and standardising tedious WGS analysis workflows. SPANDx incorporates Burrows-Wheeler Aligner (BWA) [3,4] for read mapping alignment, SAMtools [5] for read filtering and parsing, BEDTools [6] for genetic locus presence/absence (P/A) determination, Picard (http://picard.sourceforge.net) for data filtering, the Genome Analysis Tool Kit (GATK) $[7,8]$ for base quality score recalibration, variant determination, data filtering and improved insertion-deletion (indel) calling, VCFtools [9] for single-nucleotide polymorphism (SNP) and indel matrix construction, and SnpEff [10] for variant annotation. SPANDx has been written to analyse data generated from paired- and single-end Illumina (both pre- and post-v1.8 quality encoding) platforms, as well as Ion PGM and 454 singleend data.

SPANDx also incorporates several additional features aimed at minimising researcher hands-on time whilst enabling customisability. Most notably, SPANDx automatically generates a human-readable P/A matrix from individual BEDTools outputs, and can also construct error-corrected SNP and indel matrices when specified. These outputs enable quick and facile visualisation of genetic variants across a large number of genomes. SNP matrices generated by SPANDx are provided in .nex format and are directly importable into PAUP*, PHYLIP or RAxML for downstream phylogenetic analysis. Inbuilt, pre-optimised and customisable variant calling parameters for Illumina and Ion PGM data obviate the need for timeconsuming optimisation of these settings, a requirement of other programs (e.g. Galaxy [11]). Unlike many WGS tools, SPANDx does not require the user to provide assembled genomes for every strain. SPANDx is run with a single command and parallelises many tasks by taking advantage of Portable Batch System (PBS) job scheduling, thereby reducing processing times for large datasets comprising tens through to thousands of genomes. Finally, SPANDx has been written in relatively simple, noncompiled, open-source code that enables users to customise the program by incorporating their preferred NGS tools (e.g. Bowtie [12] instead of BWA for read alignment), or by adding new features to its workflow.

\section{Findings \\ SPANDx description}

The SPANDx workflow is shown in Figure 1. SPANDx is a shell package written for implementation in a Linux environment using Bash. SPANDx integrates multiple freely available Linux-based programs (BWA [3,4], SAMTools [5], Picard, GATK [7,8], VCFtools [9], BEDTools [6] and SnpEff [10]) into a single pipeline for alignment, variant identification, analysis and annotation from raw NGS data derived from haploid organisms. Using data generated from our prior WGS studies [13-16], we have tested the performance of SPANDx using paired-end Illumina $\left(\mathrm{GA}_{I I x}\right.$, MiSeq and HiSeq2000) data, and single-end Ion PGM, Illumina, and 454 GS-FLX/FLX+ data. SPANDx is designed to run in a cluster environment and utilises parallel processing for the majority of the analysis pipeline. To facilitate parallelisation and appropriate resource allocation, SPANDx requires a Linux/UNIX system with PBS. The SPANDx user manual (available at: http://sourceforge.net/projects/spandx/) provides detailed information on installing, operating and where desired, customising this program.

\section{Variant identification and phylogenetic analysis}

Variant (i.e. SNP and indel) identification is a fundamental component of any haploid WGS analysis. For this study, default settings for SPANDx (as detailed in the user manual) were used to identify variants; optional settings were included as follows. The -m flag was used to construct core genome SNP matrices from the individual Escherichia coli or Haemophilus influenzae SNP .vcf files for phylogenetic reconstruction. The SPANDx-generated Ortho_SNP_matrix.nex file was directly imported into PAUP* 4.0b10 [17] and used to construct maximum parsimony phylogenetic trees (Figures 2, 3 and 4). For the seven REL E. coli genomes, SnpEff was implemented (using the $-\mathrm{a}$ and $-\mathrm{v}$ flags) to annotate SNPs.

\section{Presence/absence (P/A) analysis of $E$. coli and $H$. influenzae genomes}

Defining the core (i.e. loci present in all taxa) vs. accessory (i.e. loci present in at least one taxon) genome is another fundamental application of haploid WGS. This information can be used for many purposes 


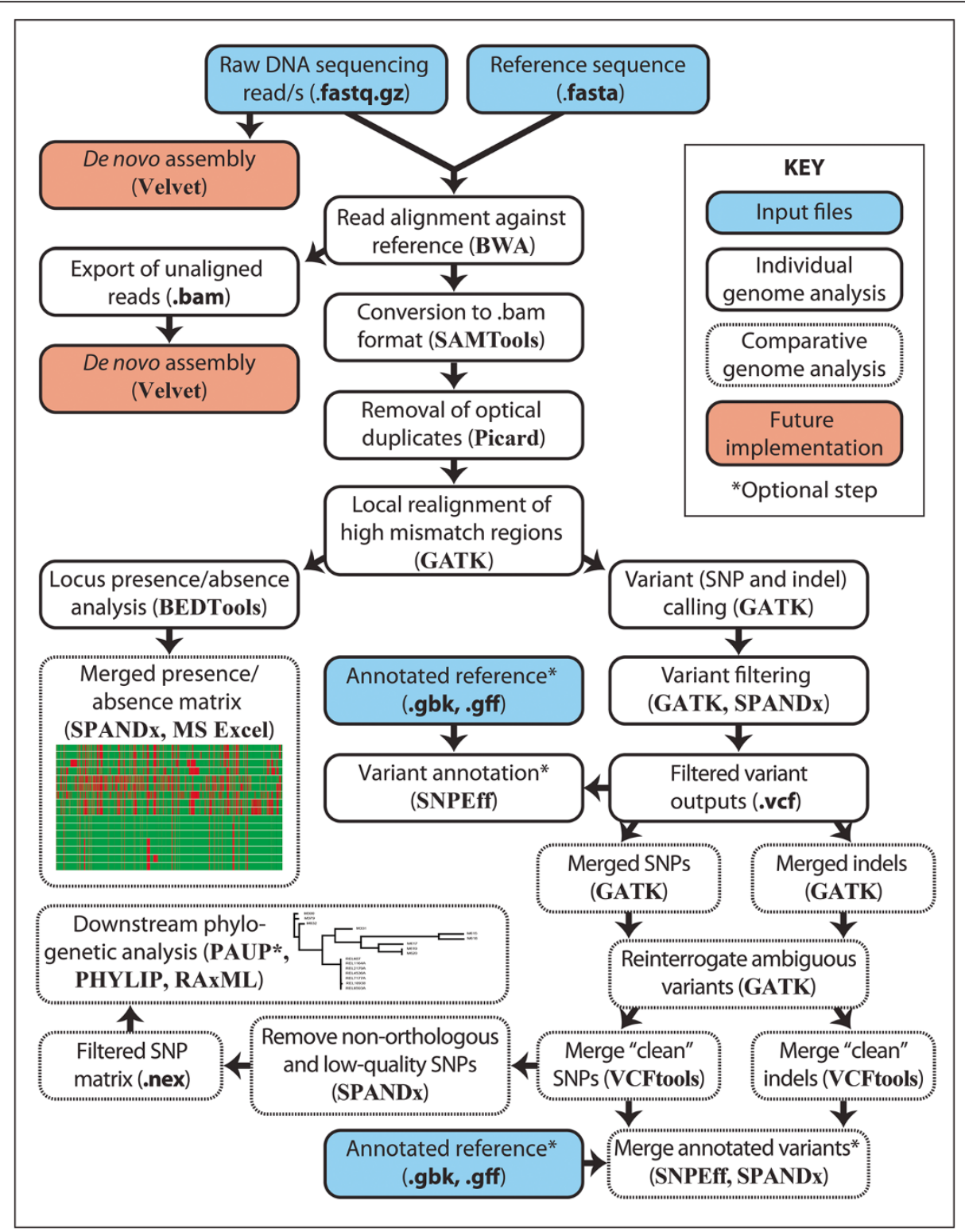

Figure 1 SPANDx workflow for analysis of haploid next-generation re-sequencing data.

including pan-genome construction, strain-, species- or genus-level signature identification, or for observing patterns of genome reduction. The coverageBED module of BEDTools has been incorporated into the SPANDx pipeline for this purpose. BEDTools determines NGS read coverage depth and breadth across segments or 'bins' relative to the reference genome [6], thereby providing an efficient way of using raw NGS reads to identify both core and accessory genomic loci within a dataset compared with a reference genome. For the current study, a default $1 \mathrm{~kb}$ window size was used for P/A analysis. SPANDx automatically generates
coverageBED genetic locus P/A outputs from all inputted genomes against the reference genome and combines individual outputs into a single human-readable matrix file (Bedcov_merge.txt). Additional file manipulation of P/A matrices was performed using basic features in MS Excel 2010 to create heat maps.

Example P/A matrices generated by SPANDx for the E. coli and $H$. influenzae datasets, which highlight the core and accessory genomes of these species compared with the reference genome, are respectively shown in Figures 3 and 4 . We have previously used these outputs to develop a novel speciation target for Burkholderia 


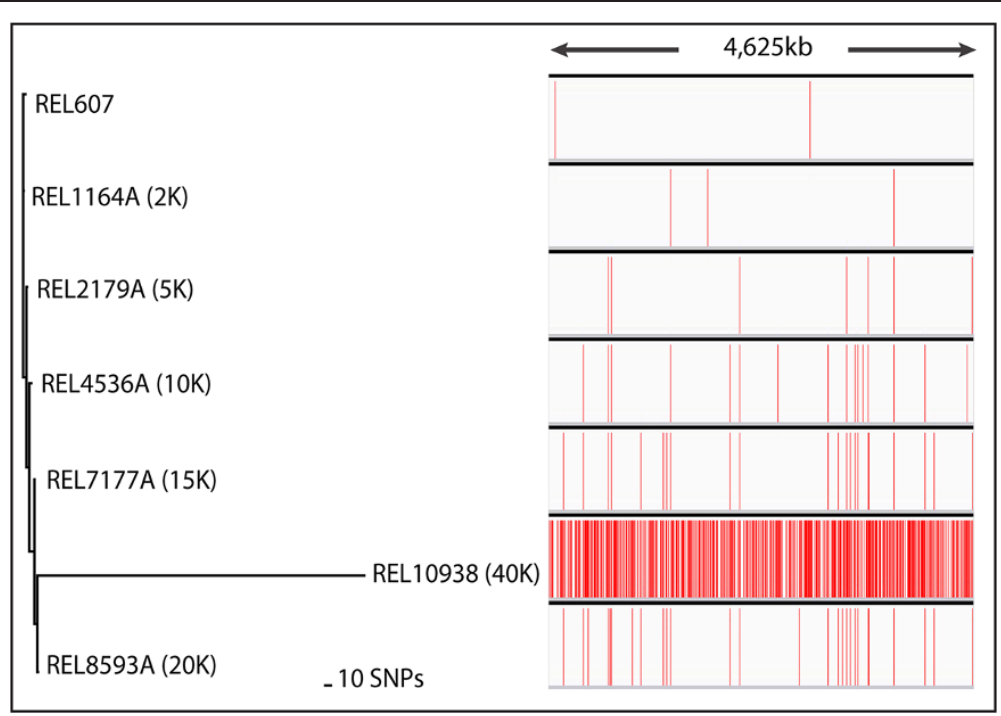

Figure 2 Single-nucleotide polymorphism (SNP) variants identified by SPANDx across the genomes of seven clonal long-term $E$. coli in vitro passaged cultures. The number of generations is indicated in parentheses. REL606, the ancestor for these passaged cultures, was used for reference genome comparison [GenBank:NC_012967]. As confirmed by SPANDx, REL607 is known to differ from REL606 by two SNPs [18], as denoted by the red vertical lines. In contrast, the $\sim 40 \mathrm{~K}$ strain REL10938 is a hypermutable strain [19] and SPANDx identified 607 SNPs separating REL10938 from REL606. Phylogenetic analysis was performed using the Ortho_SNP_matrix.nex file, an output from SPANDx that can be directly imported into PAUP* 4.0 [17]. Using maximum parsimony, a highly accurate tree (consistency index $=1.0$ ) was generated in PAUP*. SNPs were visualised with Integrative Genomics Viewer v2.3.25 [20].

ubonensis [13] and to characterise genome reduction in Burkholderia pseudomallei [14].

\section{Optimised variant calling for Illumina and Ion PGM data}

Other NGS-based genomics tools such as Galaxy [11] require users to specify variant calling settings, which can be a subjective and time-consuming task, particularly for users unfamiliar with NGS data. To combat this issue, SPANDx includes pre-optimised variant calling for both single- and paired-end haploid NGS data across the Illumina and Ion PGM platforms. Although these settings have been optimised using our test datasets, they can be customised if desired by altering the filtering parameters in GATK.config, a file that comes with the SPANDx distribution.

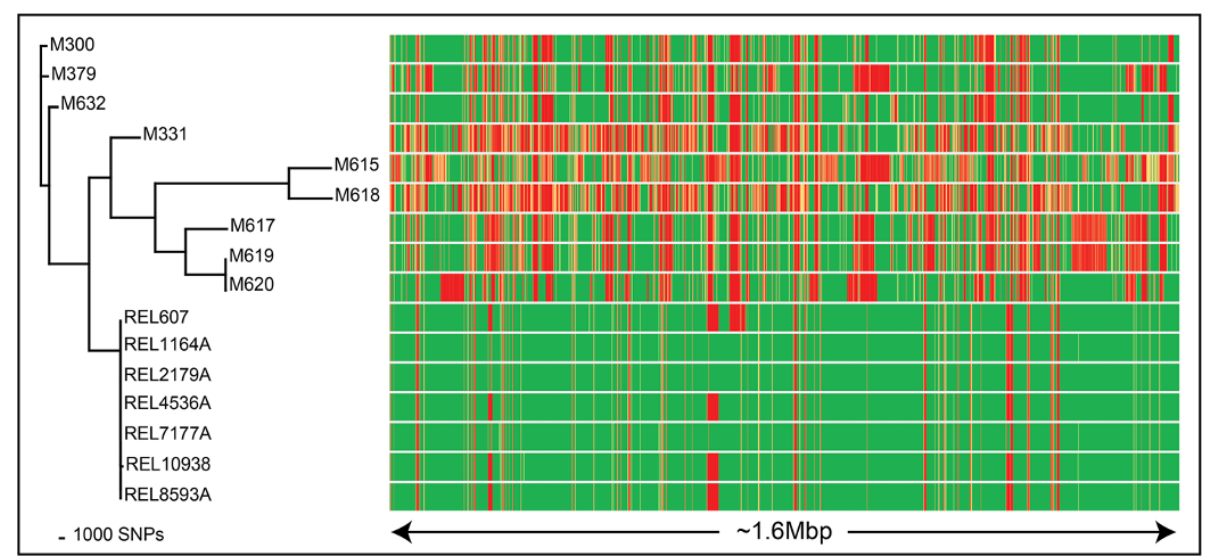

Figure 3 Core single-nucleotide polymorphism (SNP) phylogenetic analysis across 16 E. coli genomes (left), and comparison with the accessory genome (right). The Ortho_SNP_matrix.nex file created by SPANDx was directly imported into PAUP* 4.0 and used for phylogenetic construction based on 106,557 core SNPs. Using maximum parsimony, a tree with a consistency index of 0.78 was generated. The Bedcov_merge. txt file for presence/absence analysis of loci was automatically generated by SPANDx using the coverageBED module of BEDTools [6], based on the default $1 \mathrm{~kb}$ window size. Regions with <95\% coverage across one or more genomes are displayed, representing $\sim 1.6 \mathrm{Mbp}$ of the E. coli genome ( $x$-axis). Coverage is shown as a heat map, with red lines equating to low or no coverage through to green lines, which represent uniform coverage at each $1 \mathrm{~kb}$ window. In combination, these tools enable facile visualisation of the core and accessory haploid genomes. 


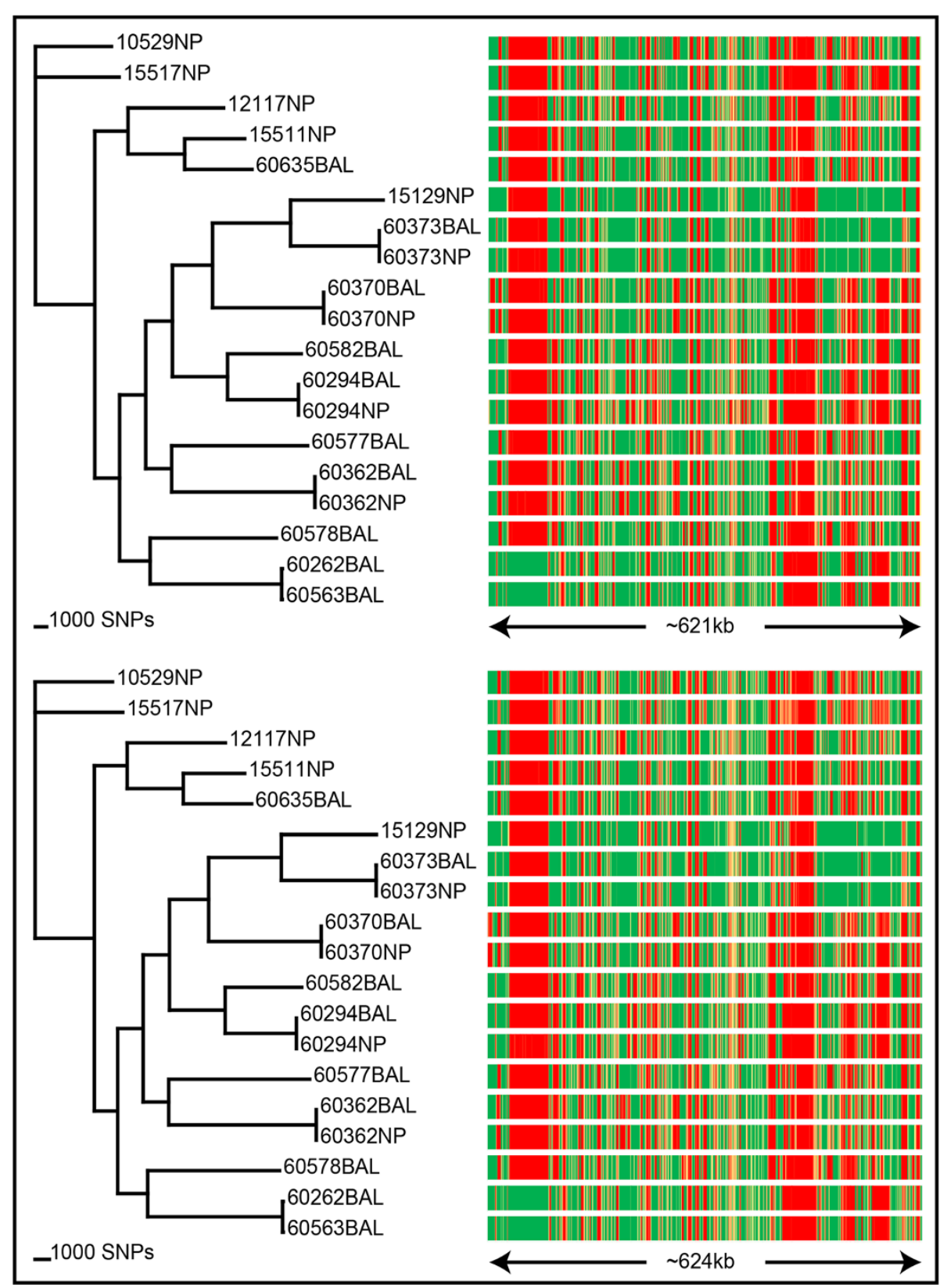

Figure 4 Comparison of Illumina and Ion PGM platforms using SPANDx. SPANDx was tested on 19 Australian Haemophilus influenzae strains [16] with both single-end lon PGM and paired-end Illumina data. Strain 86-028NP [21] was used for reference alignment. From the Illumina data (top left), $\sim 161,000$ identified SNPs were used to construct a core genome SNP cladogram $(\mathrm{Cl}=0.47)$. From the lon PGM data (bottom left), 129,000 identified SNPs were used to construct a core genome SNP cladogram $(\mathrm{Cl}=0.48)$. The right-hand side panels show corresponding presence/absence data for each strain as described in Figure 3. For Illumina, $621 \mathrm{~kb}$ was found to be variable, compared with $624 \mathrm{~kb}$ with the lon PGM data. Collectively, this comparison shows that SPANDx provides highly consistent haploid comparative genomic outputs across multiple NGS platforms.

\section{Phylogenetic analysis of SPANDx SNP outputs}

Using a combination of VCFtools, GATK and several quality control and filtering steps, SPANDx automatically generates error-corrected core genome SNP matrices for phylogenetic analysis that can be directly imported into the phylogenetic programs PAUP*, PHYLIP and RAxML, the latter two of which are open- source software. The extensive error checking, filtering and variant identification steps undertaken in SPANDx using GATK ensure that the identified SNPs are as accurate as possible using NGS data. Example maximum parsimony analyses of SPANDx-generated data for the E. coli and H. influenzae datasets are shown in Figures 2, 3 and 4. 


\section{Program comparison: SPANDx vs. BRESEQ}

We performed an in-depth comparison of SPANDx with BRESEQ, a comparative genomics tool specifically designed for identifying SNPs, indels and large deletions in closely related microbial-sized genomes (http://barricklab.org/twiki/ bin/view/Lab/ToolsBacterialGenomeResequencing). Due to limitations on BRESEQ data inputs, we only compared the six closely related $E$. coli REL genomes spanning $2 \mathrm{~K}$ to $40 \mathrm{~K}$ generations; REL607 was not included in the BRESEQ study [19] and was therefore excluded in this comparison. Default settings for SPANDx (as detailed in the SPANDx user manual) were used to identify variants. SnpEff was implemented using the $-\mathrm{a}$ and $-\mathrm{v}$ flags in SPANDx to annotate SNPs.

\section{SNPS}

SPANDx and BRESEQ identified identical SNPs for the $2 \mathrm{~K}, 5 \mathrm{~K}, 10 \mathrm{~K}, 15 \mathrm{~K}$ and $20 \mathrm{~K}$ mutants $(3,9,16,22$ and 28 SNPs, respectively) [19]. One additional SNP in the $20 \mathrm{~K}$ strain, located at position 2129116 of insB-15 in REL606, was not identified by either SPANDx or BRESEQ and was only discovered by Sanger sequencing [19]. This SNP was not able to be identified from NGS read data due to the paralogous nature of the IS1 insertion sequence element in this genome. BLAST analysis of ISI in REL606 identified 27 highly related copies ( $>99 \%$ match across $100 \%$ of bases), with up to three SNPs present among the paralogues. Using NGS data, especially data harbouring relatively small insert sizes $(\sim 80$ 170 bp with this dataset), such loci cannot be accurately mapped. Therefore, the exclusion of this SNP from both the SPANDx and BRESEQ pipelines demonstrates the inherent limitations of using short-read NGS data for variant calling in large paralogous loci.

SPANDx analysis of the $40 \mathrm{~K}$ strain identified only 608 SNPs separating the hypermutable strain REL10938 from its REL606 ancestor, compared with the 626 SNPs found using BRESEQ. Closer examination found that these 18 SNPs were either not identified by SPANDx or were excluded using the default filtering parameters due to nonpolymorphic $(n=1)$ or ambiguous $(n=11)$ genotypes, or poor mapping quality and/or insufficient $(<0.5 \times$ of average) coverage $(n=6)$. The default parameters for SNP calling in SPANDx have been optimised such that the ability to identify only 'real' variants is maximised; false-positives are not tolerated with these settings, in line with GATK recommendations. Loosening of these parameters results in additional SNPs being identified, some of which may turn out to be 'real' upon confirmation with e.g. Sanger sequencing; however, the trade-off is that false-positives begin plaguing the dataset (results not shown). Given the nature of NGS data and the behaviour of NGS alignment programs, neither variant calling method is incorrect per se, but these minor differences between programs highlight the need to verify questionable SNPs from NGS data using secondary methods including manual inspection of NGS read alignments in e.g. Tablet [22], or wet laboratory-based analyses such as Sanger sequencing or allele-specific PCR.

\section{Indels and chromosomal rearrangements}

Comparison of SPANDx and BRESEQ for identifying small $(<20 \mathrm{bp})$ indels in the REL strain cohort demonstrated that both methods were identical (variants are detailed in Supplementary Table two from [19]). Neither method identified a known 1.49Mbp inversion [23].

\section{Large deletions and insertions}

Large insertions are not currently able to be detected using SPANDx. However, for highly related strains these signatures can be detected with BRESEQ, as exemplified by the identification of ten IS element insertions with BRESEQ that were not found by SPANDx. Identification of large deletions ( $>20 \mathrm{bp}$ ) showed that, on a gross level, there was good consistency between the programs. However, the size of the deletions varied between SPANDx and BRESEQ, with SPANDx overestimating deletion size for three of the five identified deletions by $\sim 0.7$ to $1.4 \mathrm{~kb}$. BLAST analysis of these regions showed that the additional sequence called as 'deleted' by SPANDx corresponded with paralogous IS element loci (results not shown). This finding was expected, being consistent with inherent read mapping difficulties across paralogous loci using short-read NGS data.

\section{Program comparison: SPANDx vs. Galaxy}

Although we did not directly test Galaxy in this study, a previous study has used this program to compare E. coli strain REL607 with REL606 [18]. SPANDx identified that REL607 is a dual-nucleotide variant of REL606 at the $\operatorname{araA}$ and $\operatorname{rec} D$ loci (Figure 2); no indels were found by either program. Thus, SPANDx confirmed previous variant findings identified using Galaxy [18].

\section{Cross-platform reproducibility of SPANDx}

The performance of bioinformatics tools across multiple NGS platforms is an important consideration for analysis reproducibility and program utility. To address this question, we tested the performance of SPANDx using $19 \mathrm{H}$. influenzae strains subjected to two different NGS platforms: single-end Ion PGM and paired-end Illumina (MiSeq and HiSeq2000). SPANDx constructed almost identical core genome SNP phylogenies with these two datasets (Figure 4) despite being generated from platforms with inherently different error profiles and chemistries. In addition, P/A determination across these 19 genomes was essentially identical with these two platforms (Figure 4). 
These data demonstrate the robustness and accuracy of SPANDx across multiple NGS platforms.

\section{Discussion}

SPANDx is a simple-to-use, high-throughput, and open source comparative genomics tool that has been developed for the integrated analysis of haploid WGS data from start to finish with minimal hands-on time. SPANDx has been written to handle multiple NGS platforms and currently can analyse single- and paired-end read data from the Illumina MiSeq/HiSeq/GA $/ I x$ platforms, and singleend data from the Ion PGM and 454 GS FLX/FLX+ platforms. Because SPANDx uses PBS resource management, it has the capability of performing both single-core and parallel task processing, resulting in rapid turn-aroundtime, especially for medium- to large-scale WGS datasets comprising one, ten or even thousands of genomes.

SPANDx integrates existing, freely available comparative WGS analysis tools (BWA, Picard, the GATK, SAMTools, SnpEff, BEDTools and VCFtools) into a single pipeline. Importantly, SPANDx incorporates novel features for comprehensive analysis of raw haploid WGS data, and is aimed at simplifying downstream analysis (Figure 1) and increasing the user friendliness of data outputs. First, SPANDx automatically constructs P/A matrices of genetic loci using raw outputs generated by the coverageBED module of BEDTools. This feature enables identification of the core genome, a common goal of comparative haploid genome analyses. We have used this tool to design highly accurate species-specific assays for B. ubonensis [13], H. influenzae and Haemophilus haemolyticus (Price et al., manuscript in prep.), based on the identification of highly conserved loci that are absent in other species. Second, SPANDx can construct annotated, merged SNP and indel matrices from .vcf outputs. When a SNP matrix is generated, SPANDx will generate PAUP*, PHYLIP or RAxML-compatible outputs for downstream phylogenetic analysis (e.g. Figures 2, 3 and 4). Third, SPANDx contains pre-optimised yet customisable variant calling parameters for Illumina and Ion PGM data by default, allowing users to run analyses without spending a large amount of time optimising these parameters. These novel features of SPANDx enable users to quickly compare genomic data outputs without cumbersome and time-consuming manipulation of variant outputs.

Existing open-source comparative genomic tools for haploid NGS data analysis include Galaxy and BRESEQ. Galaxy (http://galaxyproject.org/) is a popular NGS tool that does not require any knowledge of Linux. The webbased version of Galaxy is particularly useful for smallscale analyses. Other advantages of Galaxy include its standardised outputs, frequent developer updates, cloudbased computer resource availability, and the ability to install the program locally where data privacy is of concern. The main limitation of Galaxy is the hands-on time required to construct an analysis pipeline, especially the need to manually optimise the filtering and data processing steps.

BRESEQ [19] is a command line tool implemented in $\mathrm{C}++$ and $\mathrm{R}$ that is useful for finding variants (SNPs, indels, large deletions and new junctions supported by mosaic reads) relative to a closely related reference genome. Comparison of BRESEQ and SPANDx outputs in the current study demonstrated that both programs gave almost identical SNP and indel outputs, suggesting that both tools excel for this purpose. However, less consensus was found when identifying large deletion boundaries, with SPANDx overestimating deleted regions in 3/5 cases due to paralogous IS element loci flanking these regions, which cannot be mapped with short-read NGS data. BRESEQ has an additional advantage over SPANDx in its ability to identify larger ( $>\sim 20 \mathrm{bp}$ ) insertions, as SPANDx is not currently configured for this purpose. However, unlike SPANDx, BRESEQ is not appropriate for WGS analysis of more distantly related genomes or for medium- to large-scale datasets. Due to its lack of parallel processing, users of BRESEQ are limited to a reference genome of $<20 \mathrm{Mb}$, an average genome coverage of $<20 \times$, and $<1,000$ expected mutations, and many comparative genomic functions are yet to be incorporated into its pipeline. BRESEQ also requires considerably more hands-on time to merge variant files than SPANDx and is thus not practical to use for more than a handful of genomes.

SPANDx has other advantages over existing tools and pipelines, including error-corrected SNP and indel matrices. To minimise effort and to standardise outputs across studies, SPANDx variant calling parameters have been optimised on our bacterial NGS datasets but can be customised to the user's preference. Using default settings, we have demonstrated that SPANDx performs comparably for SNP calling across Illumina MiSeq/HiSeq2000- and Ion PGM-generated data. To the best of our knowledge, other pipelines have not been tested and validated across multiple NGS platforms.

Recognised shortcomings of SPANDx include the inability to identify SNP variation in paralogous regions, or inversions, although these issues were also identified in BRESEQ and are the result of NGS data and not an inherent shortcoming of these programs. Currently, SPANDx requires PBS to perform parallel processing and cannot be run on systems that do not possess this software. To increase the utility of SPANDx future versions will include the ability to run this pipeline with multiple resource handlers. Although SPANDx uses BEDTools for identifying large deletions, this program does not accurately pinpoint the exact positions of large deletions and further analysis is needed. SPANDx currently does not contain tools for 
identifying large insertions. For those wishing to identify chromosomal rearrangements or large ( $>20 \mathrm{bp}$ ) insertions, or to accurately characterise large deletions, it is recommended that genome assemblies are used instead of SPANDx (or similar programs).

\section{Conclusion}

The NGS era has enabled researchers to generate unprecedented amounts of genomic data, but there remains a bottleneck in analysis. Genomic analysis pipelines such as SPANDx provide a streamlined way of decoding these data without the requirement for researchers to "reinvent the wheel" or learn multiple NGS programs. SPANDx is currently written to handle only haploid resequencing datasets. However, future development of SPANDx will include the ability to use other resource handlers (e.g. SGE), de novo assembly of accessory genome components from unaligned reads, de novo and reference-assisted genome assemblies, tools for insertion and chromosomal rearrangement detection and the ability to analyse diploid NGS data e.g. the human genome.

\section{Availability and requirements \\ Project name: SPANDx \\ Project homepage: https://sourceforge.net/projects/ spandx/}

\section{Operating system: Linux}

Programming language: Bash

Other requirements: Portable Batch System (TORQUE 2.5.13), Java 1.7.0_55, Burrows-Wheeler Aligner (BWA) 0.6.2, SAMtools 0.1.19, BEDTools 2.18.2, Picard 1.105, the Genome Analysis Tool Kit (GATK) 3.0 or higher, VCFtools 0.1.11, tabix 0.2.6 and SnpEff 3.6.

License: GNU General Public License version 3.0 (GPLv3)

Any restrictions to use by non-academics: Yes. Commercial users of GATK are required to obtain a licence for use. For further information, see www.appistry.com/ gatk. As of version 3.1, GATK is open source to not-forprofit institutions only. SPANDx and all other software used by SPANDx are open source.

\section{Availability of supporting data}

Two NGS datasets were used in this study. The first dataset comprised 16 publicly available E. coli Illumina HiSeq2000generated genomes (Sequence Read Archive [SRA] accessions ERX287459, ERX287470, ERX287479, ERX287533, ERX287535 through ERX287538; ERX287540, SRX012986, and SRX012988 through SRX012993). Seven are isogenic 'REL' isolates from long-term evolution experiments (http://myxo.css.msu.edu/ecoli/) that span 40,000 in vitro generations [19] and the additional nine are other more distantly related $E$. coli genomes from the SRA database. The FASTA file for the closed E. coli genome REL606 [19] was used as the reference for variant calling and annotation. The second dataset comprised 20 Australian $H$. influenzae strains sequenced using both the Ion PGM [16] and Illumina MiSeq [16] or HiSeq2000 platforms. The FASTA file for the closed $H$. influenzae 86-028NP genome [21] was used as the reference for variant calling.

\section{Abbreviations}

NGS: Next-generation sequencing; WGS: Whole genome re-sequencing; SPANDx: Synergised Pipeline for Analysis of NGS data in Linux; SNP: Single-nucleotide polymorphism; BWA: Burrows wheeler aligner; GATK: Genome analysis tool kit; indel: Insertion-deletion; P/A: Presence/ absence (of genetic loci)

\section{Competing interests}

The authors declare that they have no competing interests.

\section{Authors' contributions}

DSS and EPP conceived of the study, designed and implemented the software, wrote the manuscript and performed all analyses. Both authors have read and approved the final manuscript.

\section{Acknowledgements}

We wish to thank the Center for Microbial Genetics and Genomics at Northern Arizona University and Charles Darwin University for providing access to high-performance computing facilities, and the Lenski laboratory at Michigan State University and the Wellcome Trust Sanger Institute for making freely available their E. coli genomes on the Sequence Read Archive database. We also thank Bart Currie and Heidi Smith-Vaughan at Menzies School of Health Research for provision of NGS data. This work was supported by the Australian National Health and Medical Research Council via award 1046812.

\section{Author details}

${ }^{1}$ Global and Tropical Health Division, Menzies School of Health Research, Charles Darwin University, PO Box 41096, Casuarina 0811, NT, Australia. ${ }^{2}$ Child Health Division, Menzies School of Health Research, Charles Darwin University, Casuarina, PO Box 41096, Darwin 0810, NT, Australia.

Received: 14 August 2014 Accepted: 27 August 2014

Published: 8 September 2014

\section{References}

1. Margulies M, Egholm M, Altman WE, Attiya S, Bader JS, Bemben LA, Berka J, Braverman MS, Chen YJ, Chen Z, Dewell SB, Du L, Fierro JM, Gomes XV, Godwin BC, He W, Helgesen S, Ho CH, Irzyk GP, Jando SC, Alenquer MLI, Jarvie TP, Jirage KB, Kim JB, Knight JR, Lanza JR, Leamon JH, Lefkowitz SM, Lei M, Li J, et al: Genome sequencing in microfabricated high-density picolitre reactors. Nature 2005, 437:376-380

2. Zhao S, Prenger K, Smith L: Stormbow: a cloud-based tool for reads mapping and expression quantification in large-scale RNA-Seq studies. ISRN Bioinformatics 2013, 2013:481545.

3. Li H, Durbin R: Fast and accurate short read alignment with Burrows-Wheeler transform. Bioinformatics 2009, 25:1754-1760.

4. Li H, Durbin R: Fast and accurate long-read alignment with Burrows-Wheeler transform. Bioinformatics 2010, 26:589-595.

5. Li H, Handsaker B, Wysoker A, Fennell T, Ruan J, Homer N, Marth G, Abecasis G, Durbin R, 1000 Genome Project Data Processing Subgroup: The sequence alignment/map format and SAMtools. Bioinformatics 2009, 25:2078-2079.

6. Quinlan AR, Hall IM: BEDTools: a flexible suite of utilities for comparing genomic features. Bioinformatics 2010, 26:841-842

7. McKenna A, Hanna M, Banks E, Sivachenko A, Cibulskis K, Kernytsky A, Garimella K, Altshuler D, Gabriel S, Daly M, DePristo MA: The genome analysis toolkit: a MapReduce framework for analyzing next-generation DNA sequencing data. Genome Res 2010, 20:1297-1303.

8. DePristo MA, Banks E, Poplin R, Garimella KV, Maguire JR, Hartl C, Philippakis AA, del Angel G, Rivas MA, Hanna M, McKenna A, Fennell TJ, Kernytsky AM, Sivachenko AY, Cibulskis K, Gabriel SB, Altshuler DA, Daly MJ: A framework 
for variation discovery and genotyping using next-generation DNA sequencing data. Nat Genet 2011, 43:491-498.

9. Danecek P, Auton A, Abecasis G, Albers CA, Banks E, DePristo MA, Handsaker RE, Lunter G, Marth GT, Sherry ST, McVean G, Durbin R, and 1000 Genomes Project Analysis Group: The variant call format and VCFtools. Bioinformatics 2011, 27:2156-2158.

10. Cingolani P, Platts A, Wang le L, Coon M, Nguyen T, Wang L, Land SJ, Lu X, Ruden DM: A program for annotating and predicting the effects of single nucleotide polymorphisms, SnpEff: SNPs in the genome of Drosophila melanogaster strain w1118; iso-2; iso-3. Fly 2012, 6:80-92.

11. Giardine B, Riemer C, Hardison RC, Burhans R, Elnitski L, Shah P, Zhang Y Blankenberg D, Albert I, Taylor J, Miller W, Kent WJ, Nekrutenko A: Galaxy: a platform for interactive large-scale genome analysis. Genome Res 2005, 15:1451-1455.

12. Langmead B, Trapnell C, Pop M, Salzberg SL: Ultrafast and memoryefficient alignment of short DNA sequences to the human genome. Genome Biol 2009, 10:R25.

13. Price EP, Sarovich DS, Webb JR, Ginther JL, Mayo M, Cook JM, Seymour ML, Kaestli M, Theobald V, Hall CM, Busch JD, Foster JT, Keim P, Wagner DM, Tuanyok A, Pearson T, Currie BJ: Accurate and rapid identification of the Burkholderia pseudomallei near-neighbour, Burkholderia ubonensis, using real-time PCR. PLoS One 2013, 8:e71647.

14. Price EP, Sarovich DS, Mayo M, Tuanyok A, Drees KP, Kaestli M, BeckstromSternberg SM, Babic-Sternberg JS, Kidd TJ, Bell SC, Keim P, Pearson T, Currie $B J$ : Within-host evolution of Burkholderia pseudomallei over a twelveyear chronic carriage infection. mBio 2013, 4:e00388

15. Podin Y, Sarovich DS, Price EP, Kaestli M, Mayo M, Hii K, Ngian H, Wong S, Wong I, Wong J, Mohan A, Ooi M, Fam T, Wong J, Tuanyok A, Keim P, Giffard PM, Currie BJ: Burkholderia pseudomallei isolates from Sarawak, Malaysian Borneo, are predominantly susceptible to aminoglycosides and macrolides. Antimicrob Agents Chemother 2014, 58:162-166.

16. Smith-Vaughan HC, Chang AB, Sarovich DS, Marsh RL, Grimwood K, Leach AJ, Morris PS, Price EP: The absence of an important vaccine and diagnostic target in carriage- and disease-related nontypable Haemophilus influenzae. Clin Vaccine Immunol 2014, 21:250-252.

17. Swofford DL: PAUP*. Phylogenetic Analysis Using Parsimony (*and Other Methods). Version 4. Sunderland, MA: Sinauer Associates; 2003.

18. Herron MD, Doebeli M: Parallel evolutionary dynamics of adaptive diversification in Escherichia coli. PLOS Biol 2013, 11:e1001490.

19. Barrick JE, Yu DS, Yoon SH, Jeong H, Oh TK, Schneider D, Lenski RE, Kim JF: Genome evolution and adaptation in a long-term experiment with Escherichia coli. Nature 2009, 461:1243-1247.

20. Thorvaldsdottir H, Robinson JT, Mesirov JP: Integrative Genomics Viewer (IGV): high performance genomics data visualization and exploration. Brief Bioinform 2013, 14:178-192.

21. Harrison A, Dyer DW, Gillaspy A, Ray WC, Mungur R, Carson MB, Zhong H, Gipson J, Gipson M, Johnson LS, Lewis L, Bakaletz LO, Munson RS Jr: Genomic sequence of an otitis media isolate of nontypeable Haemophilus influenzae: comparative study with $\mathrm{H}$. influenzae serotype $d$, strain KW20. J Bacteriol 2005, 187:4627-4636.

22. Milne I, Stephen G, Bayer M, Cock PJ, Pritchard L, Cardle L, Shaw PD, Marshall D: Using Tablet for visual exploration of second-generation sequencing data. Brief Bioinform 2013, 14:193-202.

23. Schneider D, Duperchy E, Coursange E, Lenski RE, Blot M: Long-term experimental evolution in Escherichia coli. IX. characterization of insertion sequence-mediated mutations and rearrangements. Genetics 2000, 156:477-488.

doi:10.1186/1756-0500-7-618

Cite this article as: Sarovich and Price: SPANDx: a genomics pipeline for comparative analysis of large haploid whole genome re-sequencing datasets. BMC Research Notes 2014 7:618.

\section{Submit your next manuscript to BioMed Central and take full advantage of:}

- Convenient online submission

- Thorough peer review

- No space constraints or color figure charges

- Immediate publication on acceptance

- Inclusion in PubMed, CAS, Scopus and Google Scholar

- Research which is freely available for redistribution
C Biomed Central 